\title{
Metabolic dysfunction in pulmonary hypertension: from basic science to clinical practice
}

\author{
Stephen Y. Chan ${ }^{1}$ and Lewis J. Rubin ${ }^{2}$
}

\begin{abstract}
Affiliations: ${ }^{1}$ Center for Pulmonary Vascular Biology and Medicine, Pittsburgh Heart, Lung, Blood, and Vascular Medicine Institute, Division of Cardiology, Dept of Medicine, University of Pittsburgh School of Medicine and University of Pittsburgh Medical Center, Pittsburgh, PA, USA. ${ }^{2}$ University of California, San Diego School of Medicine, La Jolla, CA, USA.

Correspondence: Stephen Y. Chan, Center for Pulmonary Vascular Biology and Medicine, Pittsburgh Heart, Lung, Blood, and Vascular Medicine Institute, Division of Cardiology, Dept of Medicine, University of Pittsburgh School of Medicine and University of Pittsburgh Medical Center, 200 Lothrop St, BST 1704.2, Pittsburgh, PA, 15213, USA. E-mail: chansyapitt.edu
\end{abstract}

@ERSpublications

Understanding metabolic pathways in $\mathrm{PH}$ provides opportunities for improved diagnostic and therapeutic options http://ow.ly/pFQb30guez6

Cite this article as: Chan SY, Rubin LJ. Metabolic dysfunction in pulmonary hypertension: from basic science to clinical practice. Eur Respir Rev 2017; 26: 170094 [https://doi.org/10.1183/16000617.0094-2017].

ABSTRACT Pulmonary hypertension (PH) is an often-fatal vascular disease of unclear molecular origins. The pulmonary vascular remodelling which occurs in $\mathrm{PH}$ is characterised by elevated vasomotor tone and a pro-proliferative state, ultimately leading to right ventricular dysfunction and heart failure. Guided in many respects by prior evidence from cancer biology, recent investigations have identified metabolic aberrations as crucial components of the disease process in both the pulmonary vessels and the right ventricle. Given the need for improved diagnostic and therapeutic options for $\mathrm{PH}$, the development or repurposing of metabolic tracers and medications could provide an effective avenue for preventing or even reversing disease progression. In this review, we describe the metabolic mechanisms that are known to be dysregulated in $\mathrm{PH}$; we explore the advancing diagnostic testing and imaging modalities that are being developed to improve diagnostic capability for this disease; and we discuss emerging drugs for $\mathrm{PH}$ which target these metabolic pathways.

\section{Introduction}

Pulmonary hypertension $(\mathrm{PH})$ is characterised by pulmonary vasculopathy with resulting elevations of pulmonary arterial pressure. Based on the current World Health Organization clinical classification system, $\mathrm{PH}$ is divided into five groups based on presumed molecular aetiologies, clinical associations and histopathology [1]. Group 1 comprises a severe form of this disease, termed pulmonary arterial hypertension $(\mathrm{PAH})$. The other groups encompass a much larger global population, reflecting a wide

This article has been revised according to the correction published in the March 2018 issue of the European Respiratory Review.

Received: Aug 222017 | Accepted after revision: Oct 282017

Support statement: This work was supported by National Institutes of Health grants HL096834, HL124021, HL138437 and TR002073, and the American Heart Association grant 14GRNT19600012 (to S.Y. Chan). Funding was also received from Actelion Pharmaceuticals Ltd, Allschwil, Switzerland (to L.J. Rubin). Funding information for this article has been deposited with the Crossref Funder Registry.

Conflict of interest: Disclosures can be found alongside this article at err.ersjournals.com

Provenance: Publication of this peer-reviewed article was sponsored by Actelion Pharmaceuticals Ltd, Allschwil, Switzerland (principal sponsor, European Respiratory Review issue 146).

Copyright OERS 2017. ERR articles are open access and distributed under the terms of the Creative Commons Attribution Non-Commercial Licence 4.0. 
variety of conditions, such as left heart disease, hypoxic pulmonary diseases, thromboembolic conditions and multifactorial aetiologies.

$\mathrm{PH}$ and particularly PAH are morbid and fatal conditions. Current diagnostic approaches rely upon invasive haemodynamic assessment, which is not readily available worldwide. Furthermore, clinical discernment of the histological development of this disease at the level of pulmonary vascular remodelling is currently not feasible in a living patient. Because of these points and others, late diagnoses are common and portend an ominous prognosis [2,3]. Advances in the treatment of $\mathrm{PAH}$ over the past two decades have resulted in clinical improvement in many patients, but PAH remains incurable. Accordingly, there is an ongoing search for new therapies and drug targets beyond the prostacyclin, nitric oxide and endothelin signalling pathways. Additionally, there are no approved drugs for treating $\mathrm{PH}$ due to left heart disease or hypoxic lung disease, which comprise the largest population of patients with $\mathrm{PH}$ worldwide.

The molecular origins of $\mathrm{PH}$ are theorised to promote remodelling of the pulmonary vasculature, characterised by hyperproliferation and increased cellular survival [4]. Over the past 15 years, metabolic dysregulation has emerged as a leading candidate in the quest to identify the molecular drivers of pathogenesis. Metabolic alterations in affected vascular and cardiac tissues of PH patients have been observed, notable even during the development of disease rather than just at the end-stages $[5,6]$. In the context of hereditary cases of PAH, genetic haploinsufficiency of the bone morphogenetic protein receptor 2 (BMPR2), a gene which is strongly associated with the pathogenesis of $\mathrm{PH}$ but with variable penetrance, has been linked to metabolic reprogramming [7]. Of particular note, in mice harbouring BMPR2 mutations, almost half of the genes that were differentially expressed in $B M P R 2^{+/-}$mutant cells compared with controls were classified into metabolic gene ontology groups [8]. Furthermore, emerging evidence has indicated a link between metabolic dysfunction and autoimmune diseases such as scleroderma [9] and infectious pathogens such as HIV [10] which predispose patients to the development of PAH.

A major tenet of the observed metabolic changes in $\mathrm{PAH}$ is the shift from oxidative phosphorylation to glycolysis, known as the Warburg effect. This phenomenon is frequently observed in tumour tissue, but has also been reported in pulmonary vasculature cells and the failing right ventricle in PAH patients. Even beyond the Warburg effect, the proliferative, anti-apoptotic and glycolytic processes seen in diseased PAH vessels demonstrate parallels with the cellular phenotypes observed in cancer [4, 11-14]. More recent studies have linked anaplerosis and glutaminolysis (anabolic pathways that promote the production of cellular biomass for highly proliferative tumour cells [15]) to the hyperproliferative state of PAH [16]. Yet, the true extent of the metabolic commonalities between PAH and cancer is not yet known. Furthermore, whether other subtypes of $\mathrm{PH}$ exhibit similar metabolic alterations to $\mathrm{PAH}$ and to cancer remains unclear. In this review, we discuss the current state of knowledge of the dysregulated metabolic mechanisms (figure 1), in part informed by parallels to cancer, that contribute to the development of $\mathrm{PH}$. In addition, we explore how such insights are shaping diagnostic testing in order to detect the disease earlier and more accurately. Finally, we discuss ongoing efforts in targeting these pathways for therapeutic benefit in $\mathrm{PH}$.

\section{Molecular insights into metabolic dysfunction in $\mathrm{PH}$ : above and beyond the Warburg effect \\ Hypoxia-inducible factor and downstream metabolic effectors relevant to the Warburg effect}

Hypoxia-inducible factor (HIF) is a transcription factor and master hypoxic regulator, controlling metabolic reprogramming in response to low oxygen levels. HIF has a well-described role in the pathogenesis of $\mathrm{PAH}$ and hypoxia-induced $\mathrm{PH}$ with probable contributions to other $\mathrm{PH}$ subtypes [17]. In all metazoan cells, exposure to low oxygen tension inhibits the proteasomal degradation of the HIF-1 $\alpha$ / HIF- $2 \alpha$ subunit via alteration of proline hydroxylation within HIF. This stabilised HIF- $1 \alpha /$ HIF- $2 \alpha$ subunit then translocates to the nucleus, heterodimerises with HIF-1 $\beta$ and binds to the promoters of hundreds of genes. Additionally, HIF-dependent processes, both directly and indirectly, are integrally related to numerous proliferative and survival genes and pathways implicated in $\mathrm{PAH}$, including p53, leptin, caveolin-1 and PTEN, among others [18]. Evidence of the pathogenic importance of HIF in PH has been derived from several animal models, as previously reviewed [19]. For example, mice with heterozygous genetic deficiencies for either the HIF- $1 \alpha$ or HIF- $2 \alpha$ subunit display resistance to the development of hypoxia-induced PH. More recently, it was reported that constitutive activation of HIF- $2 \alpha$ in pulmonary arterial endothelial cells via genetic knockout of prolyl-4 hydroxylase 2 (Egln1) resulted in profound obliterative PAH in mice [20]. In humans, HIF activation under normal oxygen tension has been observed in pulmonary vascular cells from PAH patients. Recently, a genetic variant of HIF- $2 \alpha$ has been identified that displays increased prevalence in high-altitude $\mathrm{PH}$ cattle compared with unaffected cattle [21], thus providing rare genetic evidence of the importance of HIF in the development of $\mathrm{PH}$.

Among the first HIF-responsive genes implicated in the Warburg effect in $\mathrm{PH}$ is the mitochondrial enzyme pyruvate dehydrogenase kinase (PDK). This enzyme is well established as a gatekeeper of 


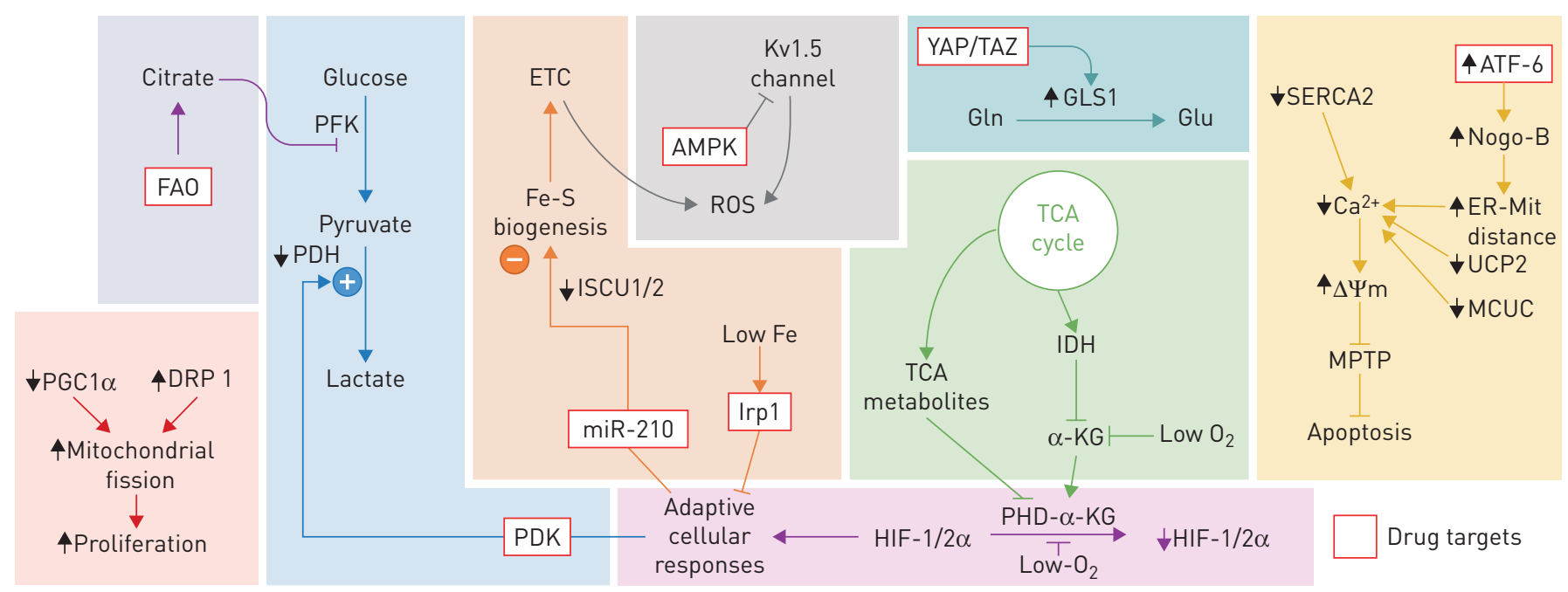

FIGURE 1 Overview of the dysfunctional metabolic pathways in pulmonary vascular cell types implicated in the development of pulmonary hypertension. KG: ketoglutarate; $\triangle \Psi \mathrm{m}$ : mitochondrial membrane potential; AMPK: adenosine monophosphate-activated protein kinase; ATF: activating transcription factor; $\mathrm{Ca}^{2+}$ : calcium; DRP: dynamin-related protein; ER: endoplasmic reticulum; ETC: electron transport chain; FAO: fatty acid oxidation; Fe: iron; S: sulfur; Gln: glutamine; GLS1: glutaminase; Glu: glutamate; HIF: hypoxia-inducible factor; IDH: isocitrate dehydrogenase; Irp: iron-regulatory protein; ISCU: iron-sulfur cluster assembly protein; Kv1.2: voltage-dependent potassium channel 1.2; MCUC: mitochondrial calcium uniporter complex; miR-210: micro-RNA 210; Mit: mitochondria; MPTP: mitochondrial permeability transition pore; Nogo-B: neurite outgrowth inhibitor-B; $\mathrm{O}_{2}$ : oxygen: PDH: pyruvate dehydrogenase; PDK: pyruvate dehydrogenase kinase; PFK: phosphofructokinase; PGC: proliferator-activated receptor-y coactivator; PHD: prolyl hydroxlase; ROS: reactive oxygen species; SERCA: sarco-/ endoplasmic reticulum calcium-ATPase; TAZ: transcription coactivator with a PDZ-binding motif; TCA: tricarboxylic acid; UCP: uncoupling protein; YAP: yes-associated protein.

oxidative metabolism, and its expression is known to be increased in response to hypoxia and in PAH [4]. Elevated levels of PDK lead to phosphorylation and inhibition of the enzyme pyruvate dehydrogenase, which in turn shunts pyruvate into glycolysis and induces the conversion of glucose to lactate by anaerobic respiration. In order to reverse the Warburg effect and thus improve $\mathrm{PH}$ manifestations, the drug dichloroacetate (DCA), an inhibitor of PDK originally developed as a cancer treatment, has been evaluated. In a number of animal models of PH, the use of DCA has demonstrated robust efficacy [22-25]. The effects of DCA in advanced human PAH have yet to be reported.

Alterations to the tricyclic acid (TCA) cycle and its intermediates can stabilise HIF. For example, $\alpha$-ketoglutarate $(\mathrm{KG})$ is a cofactor for prolyl hydroxylation and HIF degradation [26]. In addition, the TCA enzyme isocitrate dehydrogenase (IDH) has been reported to be elevated in the serum of PAH patients and in pulmonary microvascular endothelial cells derived from individuals carrying BMPR2 mutations [27]. IDH converts $\alpha-K G$ into isocitrate, with increased IDH activity leading to reduced availability of $\alpha-\mathrm{KG}$ for HIF hydroxylation. This reduces the rate of HIF degradation and increases the expression of HIF-responsive genes. Other TCA metabolites can inhibit prolyl hydroxylation and activate HIF. For example, hypoxia increases the rate at which $\alpha-\mathrm{KG}$ is reduced to 2-hydroxyglutarate (2HG), and the enantiomers $\mathrm{L} 2 \mathrm{HG}$ and D2HG can inhibit prolyl hydroxylation of HIF [28]. In human pulmonary vascular cell types, hypoxia increases $\mathrm{L} 2 \mathrm{HG}$ levels, thus controlling glycolysis and oxidative phosphorylation [29]. The influence of TCA cycle intermediates has epigenetic implications, as acetylation and methylation of nuclear histones are regulated by citrate and $\alpha-K G$, respectively $[28,30]$. Notably, the epigenetic inhibitors valproic acid and suberoylanilide hydroxamic acid (vorinostat) ameliorated $\mathrm{PH}$ in a rat model [31], supporting the concept that downstream metabolic pathways are potential therapeutic targets for $\mathrm{PH}$, at least in part.

Control of iron handling has also emerged as a key pathway implicated in HIF biology and the Warburg effect, and iron deficiency has previously been reported in $\mathrm{PAH}$ populations [32, 33]. Specifically, microRNA-210 (miR-210), a transcriptional target of HIF, was found to downregulate expression of the iron-sulfur (Fe-S) cluster assembly proteins (ISCU) 1 and 2 [34]. These are involved in the assembly of Fe-S clusters, which are prosthetic groups incorporated into enzymes involved in cellular redox signalling [35]. Hypoxic repression of ISCU1/2 via miR-210 decreased Fe-S-dependent mitochondrial respiration in favour of glycolysis in pulmonary arterial endothelial cells, thereby promoting $\mathrm{PH}$ in rodent models [36]. Importantly, a female with a genetic deficiency in ISCU1/2 was found to suffer from exercise-induced $\mathrm{PH}$, offering evidence to support a role for Fe-S clusters in the development of $\mathrm{PH}$. This relationship between 
Fe-S deficiency and PH is also supported by epidemiological data showing that histological manifestations of PAH occur in infants with a genetic deficiency in NFU1, another Fe-S cluster assembly protein [37]. More than $30 \mathrm{Fe}-\mathrm{S}$ biogenesis genes have been identified in mammalian cells [35], and it is likely that several others also contribute to the Warburg effect in $\mathrm{PH}$.

Iron can directly regulate expression of HIF-1 $\alpha$ and HIF- $2 \alpha$. Prolyl hydroxylases that regulate HIF protein stability are dependent upon iron and oxygen as cofactors. Iron deficiency decreases such hydroxylase activity and promotes HIF stability [38, 39]. In vivo, iron-deficient rats have been found to display HIF upregulation, accompanied by decreased mitochondrial activity, increased glycolytic activity and substantial pulmonary vascular remodelling. These alterations were reversed with iron replacement therapy [40]. Iron deficiency also was found to be associated with elevated hepcidin [33], which in turn can predispose to $\mathrm{PAH}$ and could serve as an additional therapeutic target. Furthermore, iron-regulatory proteins such as Irp1 are known to be influenced by both iron levels and hypoxia. Irp1-deficient mice develop PH and in pulmonary endothelial cells from these animals, increased HIF- $2 \alpha$ protein levels were observed compared with cells from wild-type animals [41]. Notably, iron-specific biology may be context-specific and/or dose-dependent, given the reported predisposition to $\mathrm{PH}$ in sickle-cell patients with iron overload [42]. Nonetheless, iron replacement therapy is currently under study as a therapy for PAH (NCT01447628), and drugs that inhibit miR-210 or Fe-S cluster biogenesis, or activate Irp1 (i.e. tempol) [43], could represent future $\mathrm{PH}$ therapies.

Independent of HIF, additional molecules have been identified that control glucose metabolism in the remodelled arteries of $\mathrm{PH}$. Peroxisome proliferator-activated receptor (PPAR) $\gamma$ is a nuclear hormone receptor and transcription factor. In pulmonary vessels, PPAR $\gamma$ is vasoprotective [44]. Furthermore, in pulmonary artery smooth muscle cells (PASMCs) from $\mathrm{PAH}$ patients and in $\mathrm{PH}$ rodents, decreased BMPR2-PPAR $\gamma$ signalling has been reported $[45,46]$ and has led to $\mathrm{PH}$ and right ventricular $(\mathrm{RV})$ hypertrophy in animals [46]. This metabolic connection of PPAR $\gamma$ with BMP signalling further correlated with studies of BMPR2 activity in regulating mitochondrial biogenesis and membrane potential, thus promoting a pro-proliferative state [7]. PPAR $\gamma$ was identified as a target of the microRNA family miR-130/ 301, a systems-level regulator of cell proliferation, vascular stiffness, vasomotor tone and metabolism [47]. Most recently, PPAR $\gamma$ was found to regulate key enzymes controlling glucose utilisation in vascular smooth muscle cells (SMCs) [48]. Despite these encouraging findings, the clinical use of older PPAR $\gamma$ agonists has been tempered by indications of adverse myocardial events [49] and has stymied advances in $\mathrm{PH}$. Nonetheless, the weight of evidence regarding the activity of PPAR $\gamma$ in $\mathrm{PH}$ indicates its potential as a future drug target, particularly for newer PPAR $\gamma$ agonists [50].

\section{Emerging metabolic and mitochondrial pathways in PH beyond the Warburg effect}

The preference for glycolysis over oxidative phosphorylation is unlikely to represent the only metabolic shift required for vascular cell proliferation in PH. Beyond the requisite ATP production, sufficient biomass must be generated to support proliferation. Anaplerosis is the replenishing of TCA carbon intermediates via either the glutaminase (GLS1)-mediated deamidation of glutamine or the carboxylation of pyruvate. In multiple subtypes of $\mathrm{PH}$, it has been reported that two transcriptional coactivators, yes-associated protein (YAP)-1 and transcriptional coactivator with a PDZ-binding motif (TAZ), are required for GLS1 upregulation and subsequent glutaminolysis to sustain vascular cell proliferation and migration within stiff pulmonary vessels [16], and is reviewed in the article by Hemnes and Humbert [51] in this issue.

TCA cycle and electron transport chain modulations are associated with alterations in reactive oxygen species (ROS), which are known to regulate pulmonary vasodilation or vasoconstriction [52]. For example, the redox-sensitive nuclear factor erythroid 2-related factor 2 (Nrf2) is a transcription factor that decreases ROS generation and subsequent inflammation. In preclinical $\mathrm{PH}$ studies, Nrf2 activation improves mitochondrial dysregulation, decreases ROS and inflammatory signalling, and consequently improves arterial and RV remodelling [53]. A chemical inducer of Nrf2, bardoxolone methyl, is under investigation in a phase II clinical study in PAH patients (NCT02036970) [54]. Although beyond the scope of this review and reviewed in detail elsewhere [55], ROS dynamics are further influenced in PH by various forms of superoxide dismutase [56], voltage gated potassium channels (Kv1.5) [57], and L-type voltage gated calcium channels, to name but a few. In this regard, Kv1.5 channels are controlled by key upstream metabolic effectors such as the AMP-activated protein kinase (AMPK). As previously reviewed, the antidiabetic drug metformin, a known stimulator of AMPK, was found to protect against the development of $\mathrm{PH}$ in both hypoxia and monocrotaline (MCT) rat models, while also displaying antiremodelling properties. Other AMPK activators, such as salicylate and methotrexate may also be effective. A clinical trial to evaluate the effects of metformin on pulmonary vascular function in patients with $\mathrm{PAH}$ is currently recruiting patients (NCT01884051). 
Mitochondrial metabolic functions depend substantially on intramitochondrial calcium dynamics. Uncoupling protein (UCP)2 is a calcium uniporter which transports calcium from the endoplasmic reticulum into mitochondria [58]. Genetic ablation of UCP2 in cultured PASMCs resulted in mitochondrial hyperpolarisation and decreased activity of calcium-sensitive mitochondrial enzymes [59, 60]. In endothelial cells, loss of UCP2 promoted mitophagy and decreased mitochondrial synthesis [61]. Correspondingly, in mice, genetic deficiency of UCP2 increased pulmonary vascular remodelling and promoted the development of $\mathrm{PH}[59,60]$. Additionally, microRNA-dependent impairment of another calcium uniporter (the mitochondrial calcium uniporter complex) resulted in decreased mitochondrial calcium levels and a concomitant PAH phenotype in PASMCs as well as in MCT rats [62]. Further downstream, calcium dynamics are dysregulated at the level of the sarco-/endoplasmic reticulum calcium-ATPase (SERCA), a sarcoplasmic reticulum transporter that is downregulated in PAH. Gene transfer of SERCA2a in both rodent and porcine PH models rescued expression of SERCA2 in pulmonary arteries, resulting in decreased pulmonary artery pressure and improved RV function [63, 64]. Additionally, dysregulated calcium homeostasis can alter electrical dynamics within the cell and mitochondria. Studies have implicated glycolysis in the control of the mitochondrial permeability transition pore, a voltage- and redox-dependent channel that remains closed under hyperpolarised mitochondrial membrane potential and thus promotes cell survival [65]. Finally, the transfer of calcium from the endoplasmic reticulum to mitochondria, specifically dependent on the protein Nogo-B, has been studied in the pulmonary vasculature and found to be important in the development of $\mathrm{PH}$ [66]. Further work will be necessary to determine whether more substantial links exist between endoplasmic reticulum stress and metabolic dysregulation in $\mathrm{PH}$.

Alterations of mitochondrial structure and biogenesis have been found to drive metabolic alterations in PH. Emerging studies have identified interconnected and dynamic sets of mitochondrial structures which exist within each cell and are controlled by an ever-changing balance of fission and fusion processes. Dynamin-related protein (Drp) 1 is a GTPase that regulates mitochondrial fission and fragmentation $[67,68]$ and has been associated with the pro-proliferative vascular state in PH [69]. Decreased levels of mitofusin-2 in PAH have also been implicated in driving mitochondrial fragmentation and an imbalance of proliferation/apoptosis [70]. Pharmacological inhibition of mitochondrial fission and Drp1 with Mdivi-1 [71, 72] has been shown to ameliorate both pulmonary vascular and right ventricular dysfunction in animal models of $\mathrm{PH}$. In parallel, decreased activation of peroxisome proliferator-activated receptor- $\gamma$ coactivator (PGC) $1 \alpha$, a transcription factor mediating mitochondrial biogenesis and fission, has been linked to $\mathrm{PH}$ [70]. Additionally, deficient BMPR2 signalling has been implicated in the control of mitochondrial fission and a pro-inflammatory state [7]. In combination with PGC1 $\alpha$, Sirtuin 3 (SIRT3), a factor implicated in the control of mitochondrial structure via protein deacetylation [73], was recently reported to be repressed in rodent PH models, and SIRT3-null mice spontaneously developed PH [74]. Yet, due to their ubiquitous activity in other organ systems, it remains to be seen whether molecules involved in controlling mitochondrial structure can be useful therapeutic targets for $\mathrm{PH}$.

\section{Dysregulated fatty acid oxidation in the diseased right ventricle}

Under non-diseased and baseline activity, fatty acid oxidation (FAO) generates $60-90 \%$ of energy production in cardiomyocytes, with the remaining 10-40\% derived from glycolysis and glucose oxidation. A mutually competitive relationship, known as the Randle cycle, exists between these processes [75]. At baseline, increased production of citrate during FAO inhibits phosphofructokinase and leads to an accumulation of glucose-6-phosphate. This inhibits hexokinase, resulting in a decrease in pyruvate production and further inhibiting glycolysis. Perhaps incited by increased pulmonary arterial pressures and impaired coronary perfusion as a result of advancing RV hypertrophy, initial RV injury in $\mathrm{PH}$ is thought to produce an inadequate oxygen supply. Consequently, HIF- $1 \alpha$ is activated in cardiomyocytes thus driving upregulation of glycolytic genes [76]. Such reprogramming consequently leads to a reduction of FAO and worsens RV hypertrophy and cardiomyocyte contractile function. In fact, the upregulation of HIF- $1 \alpha$ and glycolysis in hypertrophied RV has been demonstrated in both hypoxic and MCT PH rodent models $[77,78]$. Correspondingly, inhibition of this process in mice via administration of DCA resulted in increased cardiac output and function [79]. Targeting the Randle cycle via FAO inhibitors may improve RV function by allowing more efficient use of glucose oxidation. For example, trimetazidine and ranolazine are FAO inhibitors that enhance glucose oxidation, and both compounds improved RV function in a pulmonary artery banding model of RV failure [80]. FAO inhibitors are under investigation in clinical trials, including one with trimetazidine (NCT02102672) and a number of studies evaluating ranolazine, both published (NCT01174173) [81] and ongoing (NCT01839110, NCT02829034 and NCT01917136). Targeting dysfunction at the RV separately from dysregulation of pulmonary vascular remodelling, if used in combination with classical therapeutic approaches, may provide another avenue in the treatment of $\mathrm{PH}$. 


\section{Diagnostic application of metabolic dysregulation in $\mathrm{PH}$ Plasma metabolite signatures in $\mathrm{PH}$}

There is a need for accurate, non-invasive and early detection of $\mathrm{PH}$ and the prospect of metabolomic screening is increasingly being explored (figure 2). First, efforts have been made to determine whether metabolites in circulating blood may reflect metabolic reprogramming in the pulmonary vessels and RV. Recently, in addition to TCA intermediates and amino acids [82], alterations in RNA-based nucleosides, fatty acids, sphingomyelins, steroids and phosphatidylcholine levels have been observed in the plasma of $\mathrm{PAH}$ patients [83]. Interestingly, the largest differences in signature correlated with an increased risk of death, while correction of several metabolites over time was associated with a better clinical outcome. Plasma metabolomic analyses have been pursued in separate cohorts of patients coupled with invasive haemodynamics and radionuclide ventriculography at rest and at exercise [84]. Novel associations of right ventricular-to-pulmonary vascular (RV-PV) dysfunction with release of indoleamine 2,3-dioxygenasedependent tryptophan metabolites (IDO-TMs) into the circulation were reported. Importantly, IDO-TMs correlated with RV-PV dysfunction in a validation cohort with known risk factors for $\mathrm{PH}$ and in patients with established PAH. Interestingly, new data are emerging that show that pulmonary vasodilators can alter tissue and circulating metabolites [85-87]. If these alterations are found to correlate with therapeutic and haemodynamic benefit in $\mathrm{PAH}$, it is possible that metabolite quantification could be developed in the future as a valuable non-invasive method to monitor response to therapy. Finally, circulating microRNAs, some of which include miR-130/301 [47] and miR-210 [36] and are known to directly control metabolic reprogramming in diseased pulmonary vessels, have been identified as stably and differentially expressed in plasma of PAH patients as compared with healthy volunteers (as reviewed in [88]).

Several technical and conceptual challenges remain which block the development of circulating metabolic markers in $\mathrm{PH}$ diagnostics. First, metabolite quantitation typically requires specific expertise and standardisation of each step of the process, including plasma sampling, extraction and method of detection. The advent of a gold standard technique that is sensitive, specific and rapid is crucial for the clinical application of metabolic signatures as biomarkers. Moreover, no universal control exists to determine the relative abundance of metabolites, thus adversely affecting reproducibility of results among laboratories. In addition, it is unclear how much interindividual variation exists and to what extent the physiological context, such as exercise, time of day, diet and possibly age, may dictate variability. Moreover, many metabolites are expressed at relatively low levels, making these species difficult to assay. Finally, the majority of metabolites are ubiquitously expressed, making the source of these molecules and their role in pulmonary vascular disease more difficult to ascertain. Nonetheless, the relative stability, noninvasive sampling method and the sensitivity and specificity of quantifying metabolite signatures in $\mathrm{PH}$ are all compelling arguments for further optimisation of their use as clinical biomarkers.

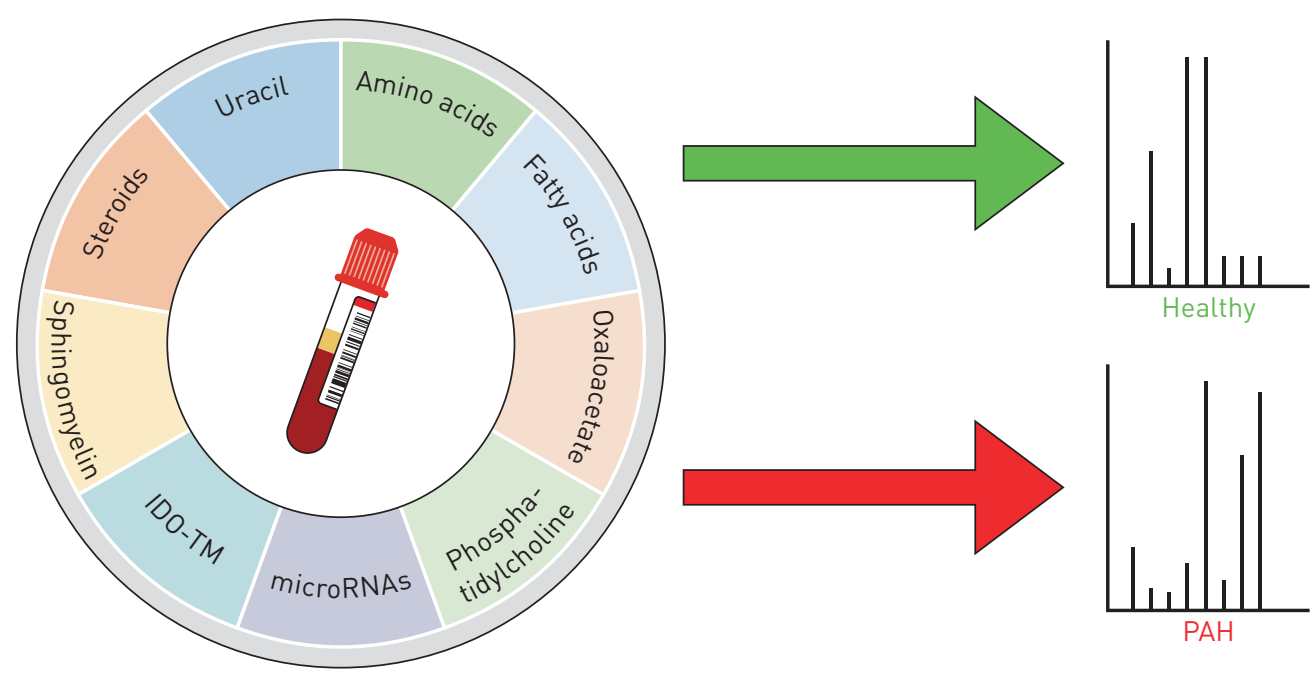

FIGURE 2 Diagnostic applications of metabolic dysregulation in pulmonary hypertension. Differences in the profiles of extracellular metabolites in circulating blood may reflect metabolic reprogramming in pulmonary arterial hypertension versus healthy individuals. IDO-TM: indoleamine 2,3-dioxygenase-dependent tryptophan metabolites; PAH: pulmonary arterial hypertension. 


\section{Metabolic imaging in $\mathrm{PH}$}

In the upcoming years, non-invasive molecular imaging is positioned to make substantial advances in pulmonary vascular disease. Positron emission tomography (PET) technology continues to expand in order to visualise the metabolic shifts occurring in $\mathrm{PH}$, such as the enhanced glucose uptake and glycolysis which occur following the inhibition of mitochondrial oxidative phosphorylation. PET can examine metabolism between two distinct anatomic compartments (the pulmonary vasculature and the RV), perhaps revealing previously undiscovered spatiotemporal relationships. The PET marker ${ }^{18} \mathrm{~F}$-fluorodeoxyglucose $\left({ }^{18} \mathrm{FDG}\right)$ a radiolabelled glucose analogue, is transported into cells and accumulates intracellularly. Highly metabolically active cells, such as those found in $\mathrm{PH}$, can then be visualised based on their ${ }^{18} \mathrm{FDG}$ levels. In the diseased pulmonary vasculature of PAH patients, PET imaging has demonstrated a chronic induction of the Warburg phenotype, as evidenced by increased glucose uptake; however, variations exist between patients $[5,89]$. The origins of imaging heterogeneity may be found in the inherent cellular heterogeneity underlying pulmonary vascular remodelling in $\mathrm{PH}$, involving multiple cell types such as endothelial cells, SMCs, fibroblasts and inflammatory cells. Thus, an increase in ${ }^{18}$ FDG uptake may be the result of both a hyperproliferative state and an invasive inflammatory component, however current limitations of ${ }^{18} \mathrm{FDG}$ PET imaging are unable to differentiate among cell types. Nonetheless, in an MCT-induced PH rat model, PET imaging demonstrated that treatment with the PDK inhibitor DCA led to lower ${ }^{18}$ FDG uptake, indicating the potential use of PET in the investigation of the biology of $\mathrm{PH}$ and in clinical applications [89].

In addition, PET imaging has shown great promise in visualising metabolic alterations of the RV. PET studies have shown substantial increases in ${ }^{18}$ FDG uptake in the RV of both animals and humans with RV hypertrophy [90] and PAH [79, 90-92] (figure 3a). More recent studies have determined that such increased $\mathrm{RV}{ }^{18} \mathrm{FDG}$ accumulation portends a poorer prognosis [97], while treatment with agents such as macitentan can attenuate such uptake concomitant with an improvement in RV function and haemodynamics [98]. PET is useful for investigations into drug distribution, target binding and drug-induced biochemical responses; however, its cost and limited availability currently restricts its use.
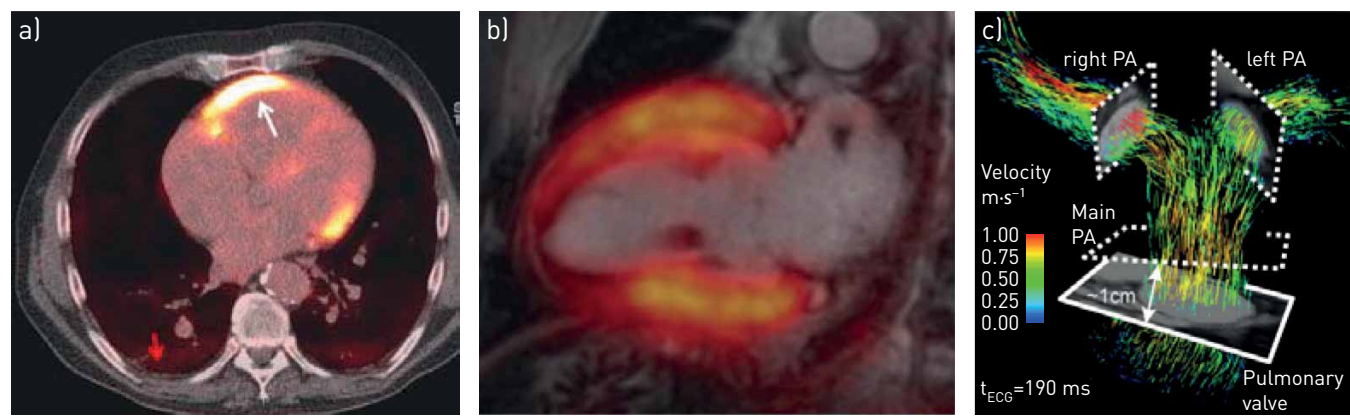

d)

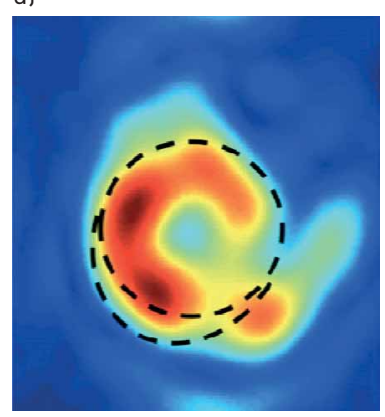

Pyruvate

\begin{abstract}
$\times 10^{7}$
\end{abstract}

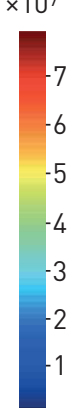

7
-6
-5
-4
-3
-2
-1

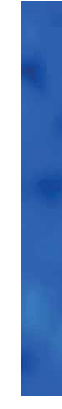

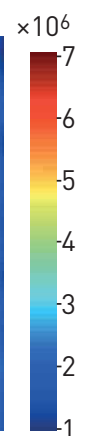

Bicarbonate

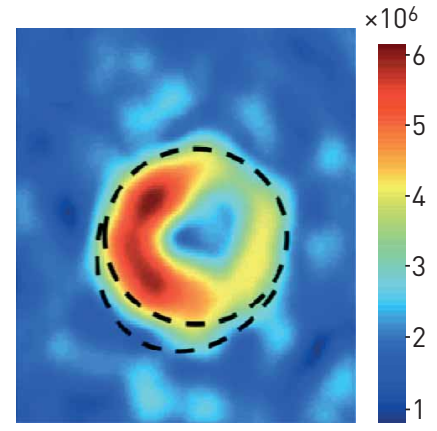

Lactate

FIGURE 3 Non-invasive molecular imaging techniques may play a future role in the management of pulmonary hypertension. a) Positron emission tomography-computed tomography shows increased ${ }^{18} \mathrm{~F}$-fluorodeoxyglucose uptake in the right ventricular wall (arrow) in pulmonary hypertension; reproduced and modified from [93] with permission. b) Magnetic resonance imaging (MRI) shows advanced structure of heart fused with positron emission tomography to determine ${ }^{18} \mathrm{~F}$-fluorodeoxyglucose uptake in areas with high metabolic activity; reproduced from [94] with permission. c) Four-dimensional flow MRI as a measure of complex three-dimensional haemodynamic changes in the pulmonary arteries; reproduced from [95] with permission. d) Cardiac hyperpolarised MRI shows the distribution of injected hyperpolarised pyruvate and its metabolic conversion to bicarbonate and lactate to visualise areas of high metabolic activity; reproduced from [96] with permission. PA: pulmonary artery. 
Furthermore, more specialised combinations of imaging, such as PET with magnetic resonance imaging (MRI) [94], may facilitate more detailed spatial resolution for metabolic dysfunction in this disease (figure 3b). Beyond PET, advances in four-dimensional flow MRI have provided an ability to assess RV kinetic energy work density and energy dissipation [95] (figure 3c). Finally, cardiac hyperpolarised MRI has been used to track specific metabolite levels in the heart and to track temporally distinct changes in pyruvate metabolism in failing human ventricles [99, 100] (figure 3d). When coupled together, such imaging modalities have the potential to provide extensive insights into the metabolic landscape of the failing RV from inception to end-stage disease, paving the way towards true precision medicine paradigms for management and treatment of this disease.

\section{Therapeutic development of metabolic drugs in $\mathrm{PH}$}

As discussed earlier and by Simmonneau et al. [101], strides are being made within the realm of metabolic intervention for $\mathrm{PH}$. However, the field is nascent, there are no approved metabolic drugs for $\mathrm{PH}$, and many challenges remain. Currently, the mainstay of investigational metabolic treatment of $\mathrm{PH}$ involves repurposing medications already approved by the US Food and Drug Administration for other diseases. Notably, because many metabolic alterations of $\mathrm{PH}$ share similarities with cancer, metabolic therapies currently being tested for a variety of cancers may have potential in PH. Repurposing drugs decreases development and approval time and could accelerate the introduction of such medications into the clinical management of $\mathrm{PH}$. However, challenges of repurposing thus far have involved issues of tissue specificity for delivery, unintended off-target effects and utilisation of drugs originally intended for acute, short-term use for more long-term therapy.

Alternatively, the development of novel small-molecule inhibitors, therapeutic antibodies or RNA-based therapies for $\mathrm{PH}$ continues to advance as new metabolic drug targets emerge (figure 1). It is likely that new, rather than repurposed medications would be necessary, particularly when targeting genetic deficiencies important in $\mathrm{PH}$ [7]. Advances in genomic, transcriptomic and metabolomic profiling offer an opportunity to individualise treatment by identifying patients with the greatest chance of response to a specific drug. The number of metabolic anomalies beyond the Warburg effect also indicates that several pathways may need to be targeted in combination for a robust clinical response. However, the most effective combination of therapies is unknown.

\section{Conclusion}

Guided by the metabolic parallels between $\mathrm{PH}$ and cancer and anchored by the Warburg effect, numerous molecular insights into $\mathrm{PH}$ pathogenesis have been reported. Yet several metabolic processes beyond the Warburg effect are emerging as integral to $\mathrm{PH}$ development, and understanding those fundamental molecular links in both the pulmonary vessels and RV will be essential for improving the clinical management of this exceptionally complex disease. Particularly exciting future directions in this field include interrogations of the molecular interconnections of metabolism with pathogenic processes such as shear stress and flow [102] as well as innate immunity [18]. In that context, the rapid innovations in molecular imaging via MRI and PET coupled with the development of metabolic tracers could provide an opportunity to individualise diagnostic and prognostic technology for $\mathrm{PH}$. Furthermore, there is hope that new metabolic drugs will emerge as a robust means for improving outcomes for $\mathrm{PH}$ patients, either singly or in combination with existing therapies.

\section{References}

1 Simonneau G, Gatzoulis MA, Adatia I, et al. Updated clinical classification of pulmonary hypertension. J Am Coll Cardiol 2013; 62: Suppl., D34-D41.

2 Chan SY, Loscalzo J. Pulmonary arterial hypertension. In: Creager MA, Beckman J, Loscalzo J, eds. Vascular Medicine: A Companion to Braunwald's Heart Disease. Philadelphia, Saunders Elsevier, 2013; pp. 667-686.

3 Humbert M, Gerry Coghlan J, Khanna D. Early detection and management of pulmonary arterial hypertension. Eur Respir Rev 2012; 21: 306-312.

4 Gurtu V, Michelakis ED. Emerging therapies and future directions in pulmonary arterial hypertension. Can J Cardiol 2015; 31: 489-501.

$5 \quad \mathrm{Xu}$ W, Koeck T, Lara AR, et al. Alterations of cellular bioenergetics in pulmonary artery endothelial cells. Proc Natl Acad Sci USA 2007; 104: 1342-1347.

6 Saygin D, Highland KB, Farha S, et al. Metabolic and functional evaluation of the heart and lungs in pulmonary hypertension by gated 2-[18F]-fluoro-2-deoxy-D-glucose positron emission tomography. Pulm Circ 2017; 7: 428-438.

7 Diebold I, Hennigs JK, Miyagawa K, et al. BMPR2 preserves mitochondrial function and DNA during reoxygenation to promote endothelial cell survival and reverse pulmonary hypertension. Cell Metab 2015; 21: 596-608.

8 Majka S, Hagen M, Blackwell T, et al. Physiologic and molecular consequences of endothelial Bmpr2 mutation. Respir Res 2011; 12: 84

9 Perl A. Metabolic control of immune system activation in rheumatic diseases. Arthritis Rheumatol 2017; doi: 10.1002/art.40223. 

a Warburg-like effect on the metabolism of HIV-infected subjects. Cytokine Growth Factor Rev 2016; 28: 1-10.

11 Rai PR, Cool CD, King JA, et al. The cancer paradigm of severe pulmonary arterial hypertension. Am J Respir Crit Care Med 2008; 178: 558-564.

12 Tuder RM, Davis LA, Graham BB. Targeting energetic metabolism: a new frontier in the pathogenesis and treatment of pulmonary hypertension. Am J Respir Crit Care Med 2012; 185: 260-266.

13 Guignabert C, Tu L, Le Hiress M, et al. Pathogenesis of pulmonary arterial hypertension: lessons from cancer. Eur Respir Rev 2013; 22: 543-551.

$14 \mathrm{Wu} \mathrm{D}$, Archer SL. Pulmonary hypertension begets pulmonary hypertension: mutually reinforcing roles for haemodynamics, inflammation, and cancer-like phenotypes. Cardiovasc Res 2016; 111: 1-4.

15 Hensley CT, Wasti AT, DeBerardinis RJ. Glutamine and cancer: cell biology, physiology, and clinical opportunities. J Clin Invest 2013; 123: 3678-3684.

16 Bertero T, Oldham WM, Cottrill KA, et al. Vascular stiffness mechanoactivates YAP/TAZ-dependent glutaminolysis to drive pulmonary hypertension. J Clin Invest 2016; 126: 3313-3335.

17 Shimoda LA, Semenza GL. HIF and the lung: role of hypoxia-inducible factors in pulmonary development and disease. Am J Respir Crit Care Med 2011; 183: 152-156.

18 Chaudhary KR, Taha M, Cadete VJ, et al. Proliferative versus degenerative paradigms in pulmonary arterial hypertension: have we put the cart before the horse? Circ Res 2017; 120: 1237-1239.

19 Cottrill KA, Chan SY. Metabolic dysfunction in pulmonary hypertension: the expanding relevance of the Warburg effect. Eur J Clin Invest 2013; 43: 855-865.

20 Dai Z, Li M, Wharton J, et al. Prolyl-4 hydroxylase 2 (PHD2) deficiency in endothelial cells and hematopoietic cells induces obliterative vascular remodeling and severe pulmonary arterial hypertension in mice and humans through hypoxia-inducible factor-2 $\alpha$. Circulation 2016; 133: 2447-2458.

21 Newman JH, Holt TN, Cogan JD, et al. Increased prevalence of EPAS1 variant in cattle with high-altitude pulmonary hypertension. Nat Commun 2015; 6: 6863.

22 Kim JW, Dang CV. Multifaceted roles of glycolytic enzymes. Trends Biochem Sci 2005; 30: 142-150.

23 McMurtry MS, Bonnet S, Wu X, et al. Dichloroacetate prevents and reverses pulmonary hypertension by inducing pulmonary artery smooth muscle cell apoptosis. Circ Res 2004; 95: 830-840.

24 Bonnet S, Michelakis ED, Porter CJ, et al. An abnormal mitochondrial-hypoxia inducible factor-1 $\alpha$-Kv channel pathway disrupts oxygen sensing and triggers pulmonary arterial hypertension in fawn hooded rats: similarities to human pulmonary arterial hypertension. Circulation 2006; 113: 2630-2641.

25 Michelakis ED, McMurtry MS, Wu XC, et al. Dichloroacetate, a metabolic modulator, prevents and reverses chronic hypoxic pulmonary hypertension in rats: role of increased expression and activity of voltage-gated potassium channels. Circulation 2002; 105: 244-250.

26 MacKenzie ED, Selak MA, Tennant DA, et al. Cell-permeating $\alpha$-ketoglutarate derivatives alleviate pseudohypoxia in succinate dehydrogenase-deficient cells. Mol Cell Biol 2007; 27: 3282-3289.

27 Fessel JP, Hamid R, Wittmann BM, et al. Metabolomic analysis of bone morphogenetic protein receptor type 2 mutations in human pulmonary endothelium reveals widespread metabolic reprogramming. Pulm Circ 2012; 2: 201-213.

$28 \mathrm{Xu} \mathrm{W}$, Yang H, Liu Y, et al. Oncometabolite 2-hydroxyglutarate is a competitive inhibitor of $\alpha$-ketoglutaratedependent dioxygenases. Cancer Cell 2011; 19: 17-30.

29 Oldham WM, Clish CB, Yang Y, et al. Hypoxia-mediated increases in L-2-hydroxyglutarate coordinate the metabolic response to reductive stress. Cell Metab 2015; 22: 291-303.

30 Wellen KE, Hatzivassiliou G, Sachdeva UM, et al. ATP-citrate lyase links cellular metabolism to histone acetylation. Science 2009; 324: 1076-1080.

31 Zhao L, Chen $\mathrm{CN}$, Hajji $\mathrm{N}$, et al. Histone deacetylation inhibition in pulmonary hypertension: therapeutic potential of valproic acid and suberoylanilide hydroxamic acid. Circulation 2012; 126: 455-467.

32 Rhodes CJ, Wharton J, Howard L, et al. Iron deficiency in pulmonary arterial hypertension: a potential therapeutic target. Eur Respir J 2011; 38: 1453-1460.

33 Rhodes CJ, Howard LS, Busbridge M, et al. Iron deficiency and raised hepcidin in idiopathic pulmonary arterial hypertension: clinical prevalence, outcomes, and mechanistic insights. J Am Coll Cardiol 2011; 58: 300-309.

34 Chan SY, Zhang YY, Hemann C, et al. MicroRNA-210 controls mitochondrial metabolism during hypoxia by repressing the iron-sulfur cluster assembly proteins ISCU1/2. Cell Metab 2009; 10: 273-284.

35 Rouault TA. Biogenesis of iron-sulfur clusters in mammalian cells: new insights and relevance to human disease. Dis Model Mech 2012; 5: 155-164.

36 White K, Lu Y, Annis S, et al. Genetic and hypoxic alterations of the microRNA-210-ISCU1/2 axis promote iron-sulfur deficiency and pulmonary hypertension. EMBO Mol Med 2015; 7: 695-713.

37 Navarro-Sastre A, Tort F, Stehling O, et al. A fatal mitochondrial disease is associated with defective NFU1 function in the maturation of a subset of mitochondrial Fe-S proteins. Am J Hum Genet 2011; 89: 656-667.

38 Ivan $\mathrm{M}$, Kondo $\mathrm{K}$, Yang $\mathrm{H}$, et al. $\mathrm{HIF} \alpha$ targeted for VHL-mediated destruction by proline hydroxylation: implications for $\mathrm{O}_{2}$ sensing. Science 2001; 292: 464-468.

39 Jaakkola P, Mole DR, Tian YM, et al. Targeting of HIF- $\alpha$ to the von Hippel-Lindau ubiquitylation complex by $\mathrm{O}_{2}$-regulated prolyl hydroxylation. Science 2001; 292: 468-472.

40 Cotroneo E, Ashek A, Wang L, et al. Iron homeostasis and pulmonary hypertension: iron deficiency leads to pulmonary vascular remodeling in the rat. Circ Res 2015; 116: 1680-1690.

41 Ghosh MC, Zhang DL, Jeong SY, et al. Deletion of iron regulatory protein 1 causes polycythemia and pulmonary hypertension in mice through translational derepression of HIF2 $\alpha$. Cell Metab 2013; 17: 271-281.

42 Wang X, Mendelsohn L, Rogers H, et al. Heme-bound iron activates placenta growth factor in erythroid cells via erythroid Krüppel-like factor. Blood 2014; 124: 946-954.

43 Ghosh MC, Tong WH, Zhang D, et al. Tempol-mediated activation of latent iron regulatory protein activity prevents symptoms of neurodegenerative disease in IRP2 knockout mice. Proc Natl Acad Sci USA 2008; 105: 12028-12033.

44 Hansmann G, Zamanian RT. PPAR $\gamma$ activation: a potential treatment for pulmonary hypertension. Sci Transl Med 2009; 1: 12ps14. 
Ameshima S, Golpon H, Cool CD, et al. Peroxisome proliferator-activated receptor gamma (PPAR $\gamma$ ) expression is decreased in pulmonary hypertension and affects endothelial cell growth. Circ Res 2003; 92: 1162-1169. Hansmann G, de Jesus Perez VA, Alastalo TP, et al. An antiproliferative BMP-2/PPAR $\gamma /$ apoE axis in human and murine SMCs and its role in pulmonary hypertension. J Clin Invest 2008; 118: 1846-1857.

47 Bertero T, Lu Y, Annis S, et al. Systems-level regulation of microRNA networks by miR-130/301 promotes pulmonary hypertension. J Clin Invest 2014; 124: 3514-3528.

48 Calvier L, Chouvarine P, Legchenko E, et al. PPAR $\gamma$ links BMP2 and TGF $\beta 1$ pathways in vascular smooth muscle cells, regulating cell proliferation and glucose metabolism. Cell Metab 2017; 25: 1118-1134.

49 Nissen SE, Wolski K. Effect of rosiglitazone on the risk of myocardial infarction and death from cardiovascular causes. N Engl J Med 2007; 356: 2457-2471.

50 Kernan WN, Viscoli CM, Furie KL, et al. Pioglitazone after ischemic stroke or transient ischemic attack. $N$ Engl Med 2016; 374: 1321-1331.

51 Hemnes AR, Humbert M. Pathobiology of pulmonary arterial hypertension: understanding the roads less travelled. Eur Respir Rev 2017; 26: 170093. Archer SL, Will JA, Weir EK. Redox status in the control of pulmonary vascular tone. Herz 1986; 11: 127-141. Eba S, Hoshikawa Y, Moriguchi T, et al. The nuclear factor erythroid 2-related factor 2 activator oltipraz attenuates chronic hypoxia-induced cardiopulmonary alterations in mice. Am J Respir Cell Mol Biol 2013; 49: 324-333.

54 Wang YY, Yang YX, Zhe H, et al. Bardoxolone methyl (CDDO-Me) as a therapeutic agent: an update on its pharmacokinetic and pharmacodynamic properties. Drug Des Devel Ther 2014; 8: 2075-2088.

55 Harvey LD, Chan SY. Emerging metabolic therapies in pulmonary arterial hypertension. J Clin Med 2017 ; 6: E43.

56 Weir EK, López-Barneo J, Buckler KJ, et al. Acute oxygen-sensing mechanisms. N Engl J Med 2005; 353: 2042-2055.

57 Remillard C, Tigno D, Platoshyn O, et al. Function of Kv1.5 channels and genetic variations of KCNA5 in patients with idiopathic pulmonary arterial hypertension. Am J Physiol Cell Physiol 2007; 292: C1837-C1853.

58 Trenker M, Malli R, Fertschai I, et al. Uncoupling proteins 2 and 3 are fundamental for mitochondrial $\mathrm{Ca}^{2+}$ uniport. Nat Cell Biol 2007; 9: 445-452.

59 Pak O, Sommer N, Hoeres T, et al. Mitochondrial hyperpolarization in pulmonary vascular remodeling Mitochondrial uncoupling protein deficiency as disease model. Am J Respir Cell Mol Biol 2013; 49: 358-367.

60 Dromparis P, Paulin R, Sutendra G, et al. Uncoupling protein 2 deficiency mimics the effects of hypoxia and endoplasmic reticulum stress on mitochondria and triggers pseudohypoxic pulmonary vascular remodeling and pulmonary hypertension. Circ Res 2013; 113: 126-136.

61 Haslip M, Dostanic I, Huang Y, et al. Endothelial uncoupling protein 2 regulates mitophagy and pulmonary hypertension during intermittent hypoxia. Arterioscler Thromb Vasc Biol 2015; 35: 1166-1178.

62 Hong Z, Chen KH, DasGupta A, et al. MicroRNA-138 and microRNA-25 down-regulate mitochondrial calcium uniporter, causing the pulmonary arterial hypertension cancer phenotype. Am J Respir Crit Care Med 2017; 195: 515-529.

63 Aguero J, Ishikawa K, Hadri L, et al. Intratracheal gene delivery of SERCA2a ameliorates chronic post-capillary pulmonary hypertension: a large animal model. J Am Coll Cardiol 2016; 67: 2032-2046.

64 Hadri L, Kratlian RG, Benard L, et al. Therapeutic efficacy of AAV1.SERCA2a in monocrotaline-induced pulmonary arterial hypertension. Circulation 2013; 128: 512-523.

65 Zamzami N, Marchetti P, Castedo M, et al. Inhibitors of permeability transition interfere with the disruption of the mitochondrial transmembrane potential during apoptosis. FEBS Lett 1996; 384: 53-57.

66 Sutendra G, Dromparis P, Wright P, et al. The role of Nogo and the mitochondria-endoplasmic reticulum unit in pulmonary hypertension. Sci Transl Med 2011; 3: 88ra55.

67 Chan DC. Mitochondrial dynamics in disease. N Engl J Med 2007; 356: 1707-1709.

68 Westermann B. Mitochondrial fusion and fission in cell life and death. Nat Rev Mol Cell Biol 2010; 11: 872-884.

69 Marsboom G, Toth PT, Ryan JJ, et al. Dynamin-related protein 1-mediated mitochondrial mitotic fission permits hyperproliferation of vascular smooth muscle cells and offers a novel therapeutic target in pulmonary hypertension. Circ Res 2012; 110: 1484-1497.

70 Ryan JJ, Marsboom G, Fang YH, et al. PGC1 $\alpha$-mediated mitofusin-2 deficiency in female rats and humans with pulmonary arterial hypertension. Am J Respir Crit Care Med 2013; 187: 865-878.

71 Parra V, Bravo-Sagua R, Norambuena-Soto I, et al. Inhibition of mitochondrial fission prevents hypoxia-induced metabolic shift and cellular proliferation of pulmonary arterial smooth muscle cells. Biochim Biophys Acta 2017; 1863: 2891-2903.

72 Tian L, Neuber-Hess M, Mewburn J, et al. Ischemia-induced Drp1 and Fis1-mediated mitochondrial fission and right ventricular dysfunction in pulmonary hypertension. J Mol Med 2017; 95: 381-393.

73 Anderson KA, Hirschey MD. Mitochondrial protein acetylation regulates metabolism. Essays Biochem 2012; 52: 23-35.

74 Paulin R, Dromparis P, Sutendra G, et al. Sirtuin 3 deficiency is associated with inhibited mitochondrial function and pulmonary arterial hypertension in rodents and humans. Cell Metab 2014; 20: 827-839.

75 Stanley WC, Lopaschuk GD, Hall JL, et al. Regulation of myocardial carbohydrate metabolism under normal and ischaemic conditions. Potential for pharmacological interventions. Cardiovasc Res 1997; 33: 243-257.

76 Graham RM, Frazier DP, Thompson JW, et al. A unique pathway of cardiac myocyte death caused by hypoxia-acidosis. J Exp Biol 2004; 207: 3189-3200.

77 Bogaard HJ, Natarajan R, Henderson SC, et al. Chronic pulmonary artery pressure elevation is insufficient to explain right heart failure. Circulation 2009; 120: 1951-1960.

78 Redout EM, Wagner MJ, Zuidwijk MJ, et al. Right-ventricular failure is associated with increased mitochondrial complex II activity and production of reactive oxygen species. Cardiovasc Res 2007; 75: 770-781.

79 Piao L, Fang YH, Cadete VJ, et al. The inhibition of pyruvate dehydrogenase kinase improves impaired cardiac function and electrical remodeling in two models of right ventricular hypertrophy: resuscitating the hibernating right ventricle. J Mol Med 2010; 88: 47-60.

80 Fang YH, Piao L, Hong Z, et al. Therapeutic inhibition of fatty acid oxidation in right ventricular hypertrophy: exploiting Randle's cycle. J Mol Med 2012; 90: 31-43. 

indices, and hemodynamic characteristics in pulmonary arterial hypertension: a pilot study. Pulm Circ 2015; 5: 547-556.

82 Bujak R, Mateo J, Blanco I, et al. New biochemical insights into the mechanisms of pulmonary arterial hypertension in humans. PLoS One 2016; 11: e0160505.

83 Rhodes CJ, Ghataorhe P, Wharton J, et al. Plasma metabolomics implicates modified transfer RNAs and altered bioenergetics in the outcomes of pulmonary arterial hypertension. Circulation 2017; 135: 460-475.

84 Lewis GD, Ngo D, Hemnes AR, et al. Metabolic profiling of right ventricular-pulmonary vascular function reveals circulating biomarkers of pulmonary hypertension. J Am Coll Cardiol 2016; 67: 174-189.

85 Sourdon J, Lager F, Viel T, et al. Cardiac metabolic deregulation induced by the tyrosine kinase receptor inhibitor sunitinib is rescued by endothelin receptor antagonism. Theranostics 2017; 7: 2757-2774.

86 Antinozzi C, Marampon F, Corinaldesi C, et al. Testosterone insulin-like effects: an in vitro study on the short-term metabolic effects of testosterone in human skeletal muscle cells. J Endocrinol Invest 2017; 40: 1133-1143.

87 Gevi F, Campolo F, Naro F, et al. The cardioprotective effect of sildenafil is mediated by the activation of malate dehydrogenase and an increase in the malate-aspartate shuttle in cardiomyocytes. Biochem Pharmacol 2017; 127: $60-70$.

88 Chun HJ, Bonnet S, Chan SY. Translational advances in the field of pulmonary hypertension. Translating microRNA biology in pulmonary hypertension. It will take more than 'miR' words. Am J Respir Crit Care Med 2017; 195: 167-178.

89 Zhao L, Ashek A, Wang L, et al. Heterogeneity in lung ${ }^{18}$ FDG uptake in pulmonary arterial hypertension: potential of dynamic ${ }^{18}$ FDG positron emission tomography with kinetic analysis as a bridging biomarker for pulmonary vascular remodeling targeted treatments. Circulation 2013; 128: 1214-1224.

90 Oikawa M, Kagaya Y, Otani $\mathrm{H}$, et al. Increased $\left[{ }^{18} \mathrm{~F}\right]$ fluorodeoxyglucose accumulation in right ventricular free wall in patients with pulmonary hypertension and the effect of epoprostenol. J Am Coll Cardiol 2005; 45: 1849-1855.

91 Can MM, Kaymaz C, Tanboga IH, et al. Increased right ventricular glucose metabolism in patients with pulmonary arterial hypertension. Clin Nucl Med 2011; 36: 743-748.

92 Fang W, Zhao L, Xiong CM, et al. Comparison of 18F-FDG uptake by right ventricular myocardium in idiopathic pulmonary arterial hypertension and pulmonary arterial hypertension associated with congenital heart disease. Pulm Circ 2012; 2: 365-372.

93 Hoesein FAM, Voortman M, Kwakkel-van Erp JM, et al. Images in COPD: combined pulmonary emphysema and fibrosis with pulmonary hypertension. Chronic Obstr Pulm Dis 2017; 4: 76-80.

94 Nensa F, Poeppel TD, Beiderwellen K, et al. Hybrid PET/MR imaging of the heart: feasibility and initial results. Radiology 2013; 268: 366-373.

95 Barker AJ, Roldán-Alzate A, Entezari P, et al. Four-dimensional flow assessment of pulmonary artery flow and wall shear stress in adult pulmonary arterial hypertension: results from two institutions. Magn Reson Med 2015; 73: 1904-1913.

96 Ball DR, Cruickshank R, Carr CA, et al. Metabolic imaging of acute and chronic infarction in the perfused rat heart using hyperpolarised $\left[1-{ }^{13} \mathrm{C}\right]$ pyruvate. NMR Biomed 2013; 26: 1441-1450.

97 Tatebe S, Fukumoto Y, Oikawa-Wakayama M, et al. Enhanced [18F]fluorodeoxyglucose accumulation in the right ventricular free wall predicts long-term prognosis of patients with pulmonary hypertension: a preliminary observational study. Eur Heart J Cardiovasc Imag 2014; 15: 666-672.

98 Drozd K, Ahmadi A, Deng Y, et al. Effects of an endothelin receptor antagonist, macitentan, on right ventricular substrate utilization and function in a Sugen 5416/hypoxia rat model of severe pulmonary arterial hypertension. $J$ Nucl Cardiol 2016; 24: 1979-1989.

99 Rider OJ, Tyler DJ. Clinical implications of cardiac hyperpolarized magnetic resonance imaging. J Cardiovasc Magn Reson 2013; 15: 93.

100 Schroeder MA, Lau AZ, Chen AP, et al. Hyperpolarized ${ }^{13} \mathrm{C}$ magnetic resonance reveals early- and late-onset changes to in vivo pyruvate metabolism in the failing heart. Eur J Heart Fail 2013; 15: 130-140.

101 Simonneau G, Hoeper MM, McLaughlin V, et al. Future perspectives in pulmonary arterial hypertension. Eur Respir Rev 2016; 25: 381-389.

102 Happé CM, Szulcek R, Voelkel NF, et al. Reconciling paradigms of abnormal pulmonary blood flow and quasi-malignant cellular alterations in pulmonary arterial hypertension. Vascul Pharmacol 2016; 83: 17-25. 